\title{
Various Techniques Used in Wireless Sensor Networks for Data Transfer
}

\author{
Ankit Saxena ${ }^{1}$, Sandeep Kumar², Swapnesh Taterh ${ }^{3}$ \\ ${ }^{1 \& 2}$ Assistant Professor, Dept. of CSE, Tula's Institute, Dehradun, Uttarakhand, India \\ ${ }^{1}$ ankit.saxena5@y ahoo.com, ${ }^{2}$ sandeepkumarsiet@gmail.com \\ ${ }^{3}$ Associate Professor, AIIT, Amity University Jaipur

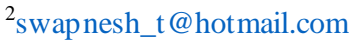

\begin{abstract}
Nowadays the wireless sensor networking is the common way for data transfer. So, it is a big task to upload and download data securely without any data loss. As we know in the wireless sensor network there are several nodes which are used to transfer the data. To perform this task there are various data transport protocols available. In this paper, we analyze the various protocol which is used for data transfer in WSN. We also discuss the various challenges which are faced during the data transfer in WSN.

Keywords- Wireless sensor network, Challenge in WSN for data transfer, protocols for data transfer in WSN.
\end{abstract}

\section{INTRODUCTION}

A Wireless Sensor Network is one kind of wireless network consist of a large number of circulating, selfdirected, low powered devices named sensor nodes which is also known as motes. These networks certainly cover a huge number of spatially distributed, little, battery operated, embedded devices that are connected to the network. Nodes are the small computers, which work jointly to form the networks. Now a days everywhere is a trend of using the wireless network. As we know it easy to setup and easy to maintain. Now in the various area the WSN is widely used as in monitoring the crowd or any sensitive area. It is also used for tracking the suspect or location finding, the CCTV and the many more. In the wireless sensor network the important role is played by the node as every node is not a normal node it is a sensor node. The sensor node is consisting of three parts

\section{Sensor Unit}

A sensor is device that senses physical phenomenon such as pressure, motion, speed etc and transform it into analog signal using a transducer [1]. A WSN integrates a large number of sensor nodes with each node containing one or more sensors depending on the application area. There is a variety of sensor types that can be employed in WSNs

\section{Transceiver Unit}

This unit is used to transfer data from one node to another. It takes care of the of the all subcomponents of the sensor node and processor and also to establish node to node interaction.[1] The transceiver plays the important role as it operates and control both the transmitter and receiver.

\section{Processing Unit}

This unit is responsible to monitor the energy consumption as well as the computational capabilities of a node. In the processing unit we use various types of process according our need some of them are microcontroller, FPGAs [1], signal processor. The most commonly processor used is microcontroller as it is simple and cheap. We also use a multi-tasking operating system.

\section{Power Supply Unit}

This unit is responsible for providing power to the sensor node. Generally, battery is the main source for power supply.[1] A battery can be recharged or replaced easily.

\section{ADVANTAGES OF WIRELESS SENSOR NETWORK}

$\checkmark$ A network can be increase or decrease without disturbing whole network.

$\checkmark \quad$ It can be setup easily

$\checkmark$ Easy to maintain.

$\checkmark \quad$ It can be used in any area such as river, sea, mountains etc.

$\checkmark \quad$ Less risk of network devices damage as there is no concept of wires. 


\section{WIRELESS SENSOR NETWORKING} APPLICATIONS

$>$ Area monitoring.

$>$ Society monitoring

$>$ Land slide detection

$>$ Home Applications

$>$ CCTV Monitoring

$>$ Air Pollution Monitoring

$>$ Water quality Monitoring

$>$ Health monitoring

$>$ Military applications

$>$ Radar

\section{WIRELESS SENSOR NETWORK} CHALLENGES

The main challenge in the wireless network is to stop the packet loss. It is responsibility of a network to ensure that data must be transfer in the network smoothly and continuously. The packet los may be occur due to EMI/RFI, congestion, transmission error or energy depletion problem. The network faces congestion problem due to flooding, whose main cause is that when all the node sends their data to the sink node. So, total data at sink node is in wide amount which cause flooding. [2.3] The reliability of the network can be measure by packet arrival probability and energy efficiency. Reliability can be classified into four types packet reliability, event reliability, upstream reliability and downstream reliability.

\section{DATA TRANSFER IN WIRELESS SENSOR NETWORKS}

Transport layer is responsible for deliver reliable data in a timely manner with no congestion and no loss of data with limited energy consumption. Transport layer protocols namely Traditional TCP and UDP are not suitable for WSN. TCP uses end to end transmission control mechanism and the congestion mechanismused is also not suitable for WSN. UDP does not support acknowledgment schemes. UDP is connectionless and does not provide flow and congestion control. [3] However, transport protocols can be made suitable for WSN by taking into account the following design considerations:

1. WSN needs a mechanism for packet loss recovery such as acknowledgement.

2. The initial connection establishment procedure should be simplified. The three ways handshake process will be a big overhead to transfer small

volume of sensed data.
3. The protocols should implement proper congestion avoidance schemes.

4. The protocols should guarantee fairness for all sensor nodes.

5. The transport control protocol should provide cross layer optimization.

Data transmission in the network can be either endto- end or on a hop-by-hop. When data is transfer by endto-end transmission mechanism, the source and the destination are responsible for the delivery of the entire packet. In case of data loss, the source has to transmit the entire packet. This leads to more energy consumption which is not feasible for wireless sensor networks. And When data is transfer Hop-by- hop transmission, it requires the neighbouring nodes to transmit the data. So, in case of data loss, retransmission can be performed easily with less energy consumption. Hop-by-hop requires local buffer and is more effective in case of multi hop WSN. Therefore hop-by-hop is more suitable for resource constrained WSN.

Reliability is achieved either by retransmission or redundancy. In retransmission mechanisms, the source node after sending the data requires an acknowledgment of its data packet from the receiver to ensure reliability. Redundancy mechanisms on the other hand send multiple copies of the same message to its receivers.

\section{Various Acknowledgment schemes used in Retransmission Mechanisms}

The following acknowledgment schemes are used to verify that the data is secure and completely transfer in wireless sensor network.

\section{Explicit acknowledgment (EACK)}

In this scheme, the receiver node sends a special control message to the source after the successful arrival of each packet. It results in high transmission overhead as acknowledgment is needed for every sent packet.

\section{Negative acknowledgment (NACK):}

In this scheme, the receiver acknowledges the sender about the missing packets.

\section{Implicit acknowledgment (IACK):}

In this scheme, the packet uses the broadcast nature of the wireless channel. The sender after sending the data listens to the channel and makes sure that the data packet is sent by the next hop to its neighbour which is a sign of acknowledgment and so on. 


\section{Selective acknowedgment (SACK):}

In this scheme, Only the lost packet of the entire message is resent again and again, while not received the acknowledgment.

\section{Retransmission mechanisms for Transport Control Protocols}

Various protocols are used for reliable transport of data. Some provide reliability in data transfer upstream direction and some in transfer data in downstream direction. Only very few protocols provide reliability in both directions. Some protocols are concentrated only on reliability, while some protocols are relay both on reliability and congestion mechanism. To provide reliable data flow, it is essential that the data path is free from congestion. All the protocols use retransmission-based data recovery in case of packet loss.

\section{Commonly Used Data Transport Protocols PSFQ}

PSFQ is stands for Pump Slowly Fetch Quickly is a downstream reliability protocol [4]. PSFQ protocol slowly injects packets into the network, in case of data loss, the protocol performs aggressive hop-by-hop recovery. It uses NACK scheme. This protocol does not provide congestion mechanis $m$ and does not handle single packet loss.

\section{ESRT}

ESRT stands for Event-to-Sink Reliable Transport protocol is an upstream reliability protocol which is used for transmitting event information rather than data packet in WSN. It includes congestion notification bit. The traffic of the source node is periodically monitored during reporting frequency. This self-configuring nature makes it to adapt to dynamic environment. It does not use NACK/ACK scheme. NACK leads to overhead and ultimately energy expenditure. This protocol also works even in case of multiple event detection using event id, which is used to identify the event.

\section{DST}

DST stands for Delay Sensitive Transport is an extended version of ESRT. DST focus to achieve event detection reliability at the sink. It introduces a Time Critical Event First scheduling policy. In case of TCEF, the data packet with minimum deadline is given first priority for retransmission in WSN. DST works best for single event and in case of random multiple event, the case becomes a bit complicated.

\section{GARUDA}

It is a downstream reliability protocol which uses hop-by-hop recovery mechanism. It uses NACK scheme and contains no congestion control mechanism for data transfer. It uses WFP (Wait for First Packet) pulse transmission mechanism in WSN.

ART

It stands for Asymmetric Reliable Transport Protocol is based on event and query reliability. This transport protocol works for both upstream as well as downstream that implements congestion control. It consists of a series of the nodes called essential nodes that over the whole sensing area. Few nodes called nonessential nodes are involved in transmission and congestion control.

\section{RMST}

It stands for Reliable Multi Segment Transport Protocol. It is a selective NACK based protocol. It works only for upstream. It is built on top of directed diffusion which discovers path from sensors to sink. In this protocol a timer is used to detect packet loss and is not suitable for event detection

\section{PORT}

PORT stands for Price Oriented Reliable Transport Protocol. It provides event reliability with minimum energy consumption. PORT works better than ESRT. It provides energy efficient congestion control mechanisms. It follows end-to-end communication mechanism. It does not support any packet recovery schemes. It is an upstream reliability protocol.

\section{Importance of Redundancy in Retransmission}

To achieving reliability is to use the concept of redundancy where multiple copies of the same packet are transmitted which is very helpful in case of packet loss.as if any packet loss then we have another copy so there will be no loss. Forward Error Correction (FEC) is used to correct only the lost or corrupted bits. FEC technique is used only in the physical layer of the network. Erasure code which is a class of FEC technique is used for error correction and detection in higher layers as the exact position of the lost or corrupted bits are known at these higher layers.

\section{Erasure Codes}

The sender divides the data packet into $\mathbf{n}$ fragments and $\mathbf{k}$ redundant bits are added so that the total number of bits send becomes $(\mathrm{n}+\mathrm{k})$. The receiver receives the data and 
decodes them when the received data is greater than or equal to $(\mathrm{n}+\mathrm{k})$. Erasure coding can be performed in a hopby-hop or end to end basis. In case of hop-by-hop mechanism, the encoding/decoding is performed at the intermediate nodes whereas end to end performs the encoding and decoding operations only at the source and sink.

\section{RDTS}

RDTS stands for Reliable erasure coding-based data transfer scheme. Here erasure coding is performed at each hop rather than at the source and sink. RDTS applies partial coding mechanism at each hop of the network. Partial coding mechanism reduces overhead as the encoding or decoding is performed on each hop only when there is a packet loss in the network. RDTS is very energy efficient. RDTS only suitable for upstream.

\section{FBcast}

FBcast is a downstream reliability broadcasting protocol based on FEC. FBcast uses fountain codes which are a class of the erasure codes. The technique is to send a block of encoded data to its neighbours node which can be further rebroadcasted to its neighbours node and so on. FBcast is suitable for single hop WSN. In case of multi hop WSN, an extended version of FBcast along with repeaters is used.

\section{DTSN}

DTSN is stands for Distributed Transport for Sensor Networks. It is an energy efficient transport protocol that support upstream data transmission in a reliable way. It provides full or differential reliability levels. When all the packets are necessary to be delivered to the sink, end to end technique is used. Full reliability mode uses ACK/NACK recovery schemes. Enhancement Flow is added with FEC to transfer data in blocks which results in high reliability. This protocol may not suitable when there are a number of hops between the source and the sink.

\section{REFERENCES}

[1] Waltenegus Dargie, Christian Poellabauer, Fundamentals of Wireless Sensor Networks Theory and Practice, ISBN 9780-470-99765-9, 2010.

[2] A Jangra, Swati, Richa, and Priyanka, "Wireless Sensor Network (WSN): Architectural Design issues and challenges", International Journal on Computer Science and Engineering, Vol.02, No.09, 2010, 3089-3094.

[3] K. Sohraby, D. Minoli, and T. Znati, "Wireless Sensor Networks Technology, Protocols, and Applications," John Wiley \& Sons, New Jersey, NJ, USA, 2007.

[4] M. Kosanovic, M. Stojcev, "Reliable Transport of Data in Wireless Sensor Network", PROC. 26th International
Conference on Microelectronics (Miel 2008), NIS, Serbia, 11-14 May, 2008

[5] S. Saqaeeyan and M. Roshanzadeh, "Improved Multi-Path and Multi-Speed Routing Protocol in Wireless Sensor Networks," International Journal of Computer Network and Information Security, Vol. 4, No. 2, 2012.

[6] Jia Zhao, Chunming Qiao, Raghuram S. Sudhaakar, Seokhoon Yoon, "Improve Efficiency and Reliability in Single-Hop WSNs with Transmit-Only Nodes," IEEE Transactions on Parallel and Distributed Systems, vol. 24, no. 3, pp. 520-534, March 2013.

[7] C. Baby and D. Suresh, "An Efficient Energy Savings Schemes using Adjacent Lossless Entropy Compression for WSN", International Journal of Advanced Engineering Research and Science, vol. 4, no. 7, pp. 146-150, 2017. Available: 10.22161/ijaers.4.7.23 